SLAC-PUB-15864

LCLS-II-TN-13-03

December 2013

\title{
Estimates of Power Radiated by the Beam in Bends of LCLS-II
}

\author{
K.L.F. Bane and P. Emma
}

(Dated: 17 December 2013) 


\section{INTRODUCTION}

In the injector region of LCLS-II the bunches pass through bends in the chicanes of the laser heater, of $\mathrm{BC} 1$ and $\mathrm{BC} 2$, and in the dogleg after the linac. The bunch repetition rate will be high, $1 \mathrm{MHz}$, and one would like to know the coherent synchrotron radiation (CSR) power radiated in the bends. We are particularly concerned about power that might radiate into cryomodules; however, for completeness we will perform calculations for all the bends in the laser heater, $\mathrm{BC} 1, \mathrm{BC} 2$, and the dogleg. In this note we estimate the CSR power for Gaussian (longitudinal) bunch distributions, using simple free-space and shielded CSR wake models. In the Appendix we repeat the calculations for more realistic bunch shapes obtained by numerical simulation. Finally, for completeness, we also calculate the incoherent synchrotron radiation (ISR) generated in the bends. Even though the ISR power radiated will be much less than the CSR power, it is of special interest because the spectrum reaches to much higher frequencies.

For LCLS-II the nominal bunch charge is $Q=100 \mathrm{pC}$, though cases of $300 \mathrm{pC}$ and $500 \mathrm{pC}$ are also of interest. The nominal bunch current shape (starting near the end of $\mathrm{BC} 2$ ) is a flat-top with a peak current of $1 \mathrm{kA}$. For $Q>300 \mathrm{pC}$ the bunch repetition rate $f_{\text {rep }}$ will be reduced to keep the average beam power at $1.2 \mathrm{MW}$ (the beam energy is $4 \mathrm{GeV}$ ); thus, at $500 \mathrm{pC}$, $f_{\text {rep }}=600 \mathrm{kHz}$. We will focus our calculations on the $300 \mathrm{pC}$ case, since as we

will see, it will generate the largest CSR power. The results for other beam current scenarios can easily be obtained, for Gaussian or uniform distributions, using equations shown in the text below. 


\section{CSR AND ISR POWER ASSUMING GAUSSIAN BUNCH SHAPES}

To estimate the CSR power radiated in the bends of LCLS-II, we begin with the CSR formula for a bunch moving in a circle of radius $\rho$ in free space [1]. For a sequence of Gaussian bunches, the average CSR power radiated is given by

$$
P_{c s r}=\varkappa L Q^{2} f_{r e p}
$$

with $\varkappa$ the loss factor, $L$ the length of the magnet, $Q$ the bunch charge, and $f_{\text {rep }}$ the repetition rate; the loss factor in turn is given by

$$
\varkappa=(0.758) \frac{Z_{0} c}{2 \cdot 3^{4 / 3} \pi} \frac{1}{\rho^{2 / 3} \sigma_{z}^{4 / 3}},
$$

where $Z_{0}=377 \Omega, c$ is the speed of light, and $\sigma_{z}$ is the rms bunch length. Thus we see that, for the beam currents being considered for LCLS-II, the $300 \mathrm{pC}$ case will generate the most CSR power, so this is the case we will use for our calculations.

In Table I we present beam and magnet properties in the bends of the chicanes of the laser heater, $\mathrm{BC} 1, \mathrm{BC} 2$, and the dogleg (with names beginning, respectively, with $\mathrm{BXH}, \mathrm{BX} 1, \mathrm{BX} 2, \mathrm{BX} 3)$. Note that the absolute value of the bending angle is simply given by $|\theta|=L / \rho$. In Column 8 of the table we give, for the case of bunch charge $Q=300 \mathrm{pC}$, the CSR power radiated assuming no shielding, $P_{c s r}$. We note that the maximum power is radiated in the last bend of $\mathrm{BC} 2,48.5 \mathrm{~W}$, and in the bends of the dogleg $40.1 \mathrm{~W}$.

If the beam pipe has a flat geometry and the vertical half-aperture satisfies $h \lesssim \rho^{1 / 3} \sigma_{z}^{2 / 3}$ the radiation is suppressed [1]. The CSR power radiated in the presence of shielding can be written as

$$
P_{c s r-s h}=f_{s h} P_{c s r}
$$

with $f_{s h}$ a shielding factor (with $0 \leq f_{s h} \leq 1$ ). The CSR point charge wake for this shielding case has been obtained by Murphy, Krinsky, Gluckstern [2]. We 
TABLE I. Magnet and beam properties in the chicanes and dogleg of LCLS-II: beam energy $E$, magnet length $L$, (horizontal) bending radius $\rho$, focusing function $\beta_{x}$, dispersion $\eta_{x}$, rms bunch length $\sigma_{z}$; CSR power radiated using Gaussian approximation, and assuming no shielding $P_{c s r}$ and with shielding $P_{c s r-s h}$; ISR power $P_{i s r}$. Here charge $Q=300 \mathrm{pC}$, repetition rate $f_{\text {rep }}=1 \mathrm{MHz}$, and full vertical aperture is taken everywhere to be $2 h=2 \mathrm{~cm}$.

\begin{tabular}{||l|c|c|c|c|c|c|c|c|c||}
\hline Name & $E[\mathrm{GeV}]$ & $L[\mathrm{~m}]$ & $\rho[\mathrm{m}]$ & $\beta_{x}[\mathrm{~m}]$ & $\eta_{x}[\mathrm{~m}]$ & $\sigma_{z}[\mathrm{~mm}]$ & $P_{c s r}[\mathrm{~W}]$ & $P_{c s r-s h}[\mathrm{~W}]$ & $P_{i s r}[\mathrm{~mW}]$ \\
\hline \hline BXH1 & 0.098 & 0.125 & 1.23 & 10.3 & 0.002 & 1. & 0.31 & 0.06 & 0.03 \\
BXH2 & 0.098 & 0.125 & 1.23 & 10.1 & 0.073 & 1. & 0.31 & 0.06 & 0.03 \\
BXH3 & 0.098 & 0.125 & 1.23 & 10.1 & 0.073 & 1. & 0.31 & 0.06 & 0.03 \\
BXH4 & 0.098 & 0.125 & 1.23 & 10.3 & 0.002 & 1. & 0.31 & 0.06 & 0.03 \\
BX11 & 0.25 & 0.204 & 1.98 & 25.7 & 0.003 & 1. & 0.37 & 0.03 & 0.86 \\
BX12 & 0.25 & 0.204 & 1.98 & 9.89 & 0.003 & 0.7 & 0.59 & 0.16 & 0.86 \\
BX13 & 0.25 & 0.204 & 1.98 & 6.11 & 0.272 & 0.6 & 0.73 & 0.28 & 0.86 \\
BX14 & 0.25 & 0.204 & 1.98 & 25.7 & 0.003 & 0.27 & 2.1 & 1.7 & 0.86 \\
BX21 & 1.6 & 0.55 & 10.2 & 108. & 0.004 & 0.27 & 1.9 & 0.70 & $147 .||$ \\
BX22 & 1.6 & 0.55 & 10.2 & 32.5 & 0.561 & 0.16 & 3.8 & 2.7 & $147 . \mid$ \\
BX23 & 1.6 & 0.55 & 10.2 & 25. & 0.561 & 0.12 & 5.6 & 4.6 & $147 .||$ \\
BX24 & 1.6 & 0.55 & 10.2 & 4.7 & 0.004 & 0.0238 & 48.5 & 48.2 & 147. \\
BX31B & 4. & 2.62 & 141. & 9.71 & 0.006 & 0.0238 & 40.1 & 36.0 & 143. \\
BX32B & 4. & 2.62 & 141. & 9.71 & 0.006 & 0.0238 & 40.1 & 36.0 & 143. \\
\hline \hline
\end{tabular}

have taken their formulation and written a Mathematica program to find the shielding factor $f_{\text {sh }}$ for Gaussian bunches as a function of shielding parameter $\Pi \equiv \sigma_{z} \rho^{1 / 2} / h^{3 / 2}[3]$. The results are plotted in Fig. 1 (the blue curve). The dashed curve gives a Gaussian fit to the numerical results, $e^{-(\Pi / \sigma)^{2} / 2}$, with 
$\sigma=.609$. We note that the fit is a good approximation for $\Pi \lesssim 1$.

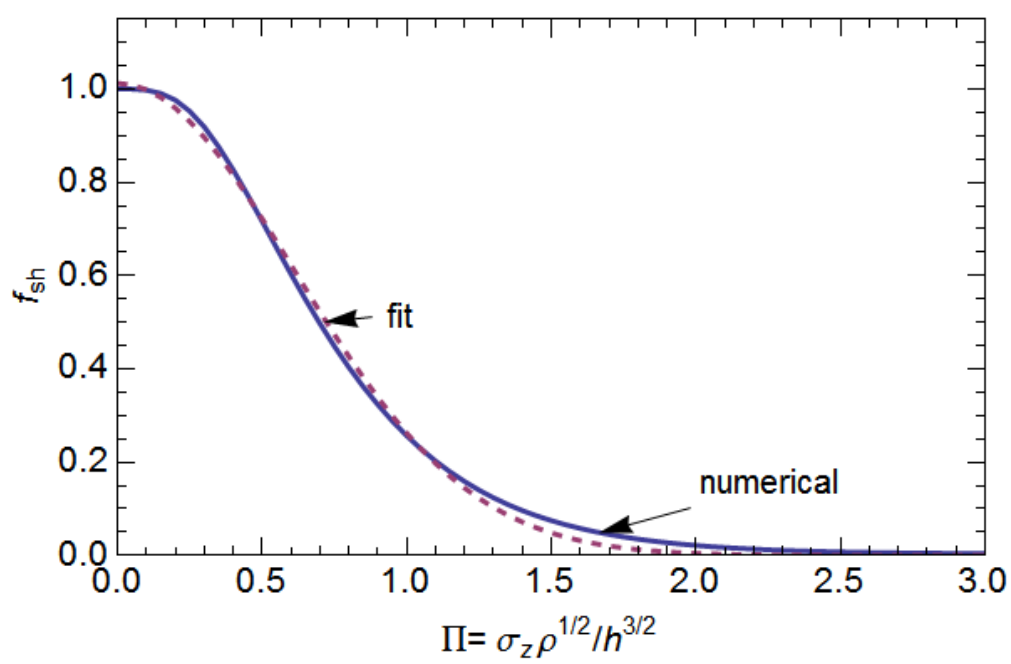

FIG. 1. The shielding factor $f_{s h}$ vs. shielding parameter $\Pi$ for a Gaussian beam moving in a circle between conducting planes separated by the distance $2 h$. The numerical results, using the Murphy, Krinsky, Gluckstern point charge wake is shown in blue. The dashed curve gives a Gaussian fit $e^{-(\Pi / \sigma)^{2} / 2}$, with $\sigma=.609$.

In Table I, in Column 9, we give the power loss for the shielded results $P_{c s r-s h}$ taking the Gaussian approximation for $f_{s h}$. Without detailed knowledge of the beam pipe apertures that will be used in LCLS-II, we have just assumed the full vertical aperture for all magnets will be $2 h=2 \mathrm{~cm}$. [Other than the first bend in BC1 (BX11) which has shielding parameter $\Pi=1.4$, all the other bends have $\Pi \leq 1.1$.] We note that in the last three bends of the table, where the CSR power is the largest, shielding has little effect. In the last bend of $\mathrm{BC} 2(\mathrm{BX} 24)$ the shielding parameter $\Pi=0.08$, and the shielding factor $f_{s h}=0.99$. 


\section{Non-gaussian Bunch Shapes}

In the region of largest CSR power loss, in the last bend of $\mathrm{BC} 2$ and the dolgleg bends, the bunch current is far from Gaussian. In BX24 of BC2 it can be approximately described as a uniform distribution with peak $\hat{I}=1.09 \mathrm{kA}$ and full width $\ell=79 \mu \mathrm{m}$. In the Appendix of this note we show that for a uniform distribution the CSR loss factor $\varkappa$ is 1.13 times that for a Gaussian with the same rms length (note that for a uniform distribution $\ell=2 \sqrt{3} \sigma_{z}$, with $\sigma_{z}$ the rms length). The power loss at BX24 becomes $P_{c s r}=54.8 \mathrm{~W}$.

The actual, numerically obtained beam shape in bend BX24 currently (tentatively) can be described as a uniform distribution with peak $\hat{I}=1.09 \mathrm{kA}$ and full width $\ell=79 \mu \mathrm{m}$, but with a long tail (see Fig. 2 in the Appendix). In the Appendix we perform, for this distribution, the CSR power calculation and find that the power radiated $P_{c s r}=42.1 \mathrm{~W}$.

One thing that we can conclude from these calculations is that the total CSR power radiated by the beam is not terribly sensitive to the details of the bunch shape.

\section{Other Bunch Charge Scenarios}

For estimating the power radiated for other bunch charge scenarios in LCLS-II, note that, if shielding is not important, the power scales as $P_{c s r} \sim$ $f_{\text {rep }} Q^{2} / \sigma_{z}^{4 / 3}$. For $Q<300 \mathrm{pC}$, the peak current remains unchanged as does $f_{\text {rep }}$. Consequently, the power will scale as $Q^{2 / 3}$. For the nominal $Q=100 \mathrm{pC}$ case the CSR power loss in BX24 becomes $23.3 \mathrm{~W}(26.4 \mathrm{~W})$ in the Gaussian (uniform) approximation. In the Appendix we calculate the CSR power radiated by the nominal bunch taking a more realistic, numerically obtained bunch shape, and find that the radiated power is $23.9 \mathrm{~W}$. 
For $300 \mathrm{pC} \leq Q \leq 500 \mathrm{pC}$, the peak current will be kept fixed, and $f_{\text {rep }}$ will be reduced to keep the average beam power at $1.2 \mathrm{MW}$. Thus the CSR power will scale as $Q^{-1 / 3}$ compared to $P_{c s r}$ for the $300 \mathrm{pC}$ case of Table I. For $Q=500 \mathrm{pC}$ the CSR power loss at BX24 is $40.9 \mathrm{~W}(46.2 \mathrm{~W})$ for the Gaussian (uniform) distribution approximation.

The CSR power loss results at BX24, for the $Q=100,300,500 \mathrm{pC}$ scenarios are summarized in Table II.

TABLE II. CSR power $P_{c s r}$, in watts, for different bunch charge scenarios radiated in the last bend of $\mathrm{BC} 2, \mathrm{BX} 24$. Results are given using a Gaussian or uniform bunch distribution approximation, or using an actual, numerically obtained charge distribution. Since the effect of shielding is negligible at this location (if we assume a full vertical aperture $2 h=2 \mathrm{~cm}$ ), the results are obtained using free-space CSR calculations.

\begin{tabular}{||c|c|c|c||}
\hline \hline Bunch Charge $[\mathrm{pC}]$ & Gaussian & Uniform & Actual \\
\hline \hline 100 & 23.3 & 26.4 & 23.9 \\
300 & 48.5 & 54.8 & 42.1 \\
500 & 40.9 & 46.2 & \\
\hline \hline
\end{tabular}

Before leaving this topic, we should point out that the peak current and bunch length in BX24 and beyond are very sensitive to the upstream linac (L2) RF phase. For example, for the $300 \mathrm{pC}$ scenario, simulations show that a $1^{\circ}$ phase error can increase the peak current and reduce the bunch length by about an order of magnitude each. A factor of 10 reduction in $\sigma_{z}$ will, according to Eq. 2, increase the radiated power by a factor of 20 , to $800 \mathrm{~W}$. Thus, the LCLS-II will need to implement diagnostics and feedback to make sure an RF phase error does not lead to an inordinate amount of radiated power reaching the cryomodules. 


\section{Incoherent Synchrotron Radiation}

Finally, for completeness we have also calculated the incoherent synchrotron radiation in the bends. The power formula is given by [4]

$$
P_{i s r}=\frac{Z_{0} c}{6 \pi} \frac{\gamma^{4}|\theta|}{\rho} e Q f_{\text {rep }},
$$

with $\gamma$ the Lorentz energy factor and $e$ the charge of an electron. For our parameters with $Q=300 \mathrm{pC}$ we find that $P_{i s r}$ radiated in $\mathrm{BC} 2$ and dogleg bends are all $0.15 \mathrm{~W}$, with much smaller losses in the bends further upstream (see Table I, Column 10).

\section{APPENDIX: THE CSR EFFECT WITH REAL BUNCH SHAPES}

The most power is radiated in bends where the bunch is the shortest, i.e. in the last bend of BC2 (BX24) and in the dogleg bends (BX31B, BX32B). In these regions the bunch shape is far from Gaussian. The current, tentative 300 pC bunch shape, obtained using LiTrack [5] simulations, can be described as a uniform distribution with a long tail [6]. It is shown in Fig. 2 by the red curve (note that the current scale is not given in the plot, and that the bunch head is to the left, at $s<0)$. The current can be approximated by a uniform

distribution of peak current $\hat{I}=1.09 \mathrm{kA}$ and full width $\ell=82.6 \mu \mathrm{m}(\mathrm{rms}$ $23.8 \mu \mathrm{m}$, the dashed curve in the figure).

We have seen in Table I that for the shortest bunches in LCLS-II shielding has almost no effect, so to obtain the loss factor for this non-Gaussian shape we use just the free-space point charge wake, given by

$$
w(s)=\frac{2}{3^{4 / 3}} H(-s) \frac{1}{\rho^{2 / 3}(-s)^{4 / 3}},
$$

with $s$ the separation between driving and test particles, where $s<0$ means the test particle is ahead of the driving particle; with $H(s)=0$ (1) for $s<0$ 


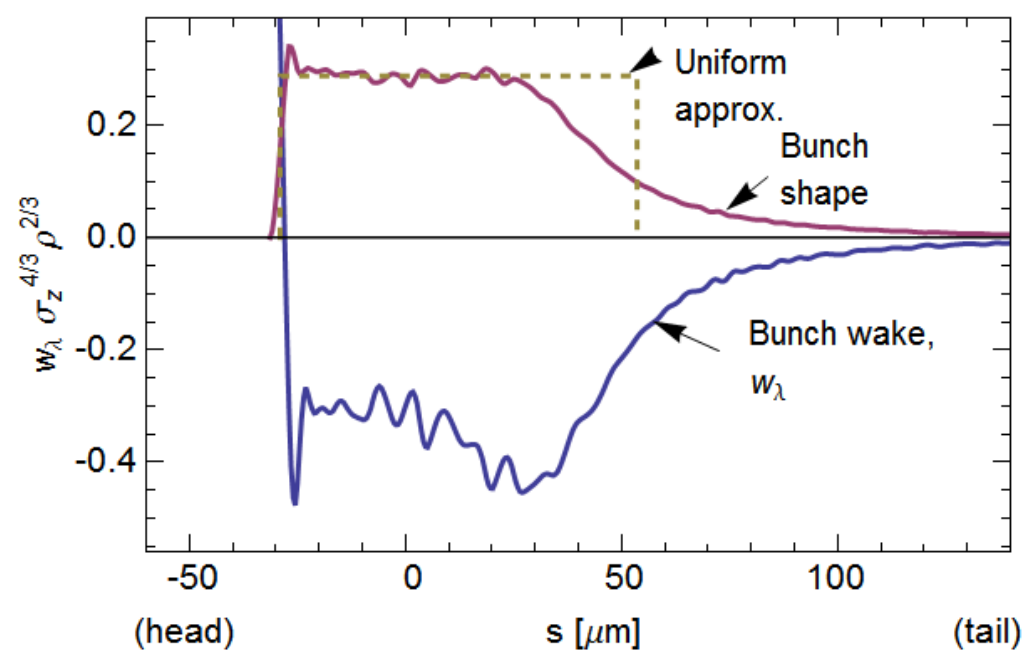

FIG. 2. For the current (tentative) $300 \mathrm{pC}$ charge distribution, the bunch wake $w_{\lambda}(s)$ (blue curve) [6]. The bunch shape is also shown, with the head to the left (the red curve). The dashed curve gives a uniform approximation, with full width $82.6 \mu \mathrm{m}$, rms length $23.8 \mu \mathrm{m}$, and peak $1.09 \mathrm{kA}$.

$(>0)$. Note that the wake at position $s$ only depends on particles behind it (at position $s^{\prime} \geq s$ ). Then the bunch wake is given by

$$
w_{\lambda}(s)=-\frac{1}{Q c} \int_{0}^{\infty} S\left(-s^{\prime}\right) I^{\prime}\left(s+s^{\prime}\right) d s^{\prime},
$$

with the step-function response

$$
S(s)=\int_{0}^{s} w\left(-s^{\prime}\right) d s^{\prime}=-\frac{2}{3^{1 / 3}} H(-s) \frac{1}{\rho^{2 / 3}(-s)^{1 / 3}}
$$

and $I^{\prime}(s)$ the slope of the bunch current distribution. The loss factor, the average voltage loss per unit charge and unit length of travel, is given by the average of the bunch wake

$$
\varkappa=-\frac{1}{Q c} \int_{-\infty}^{\infty} w_{\lambda}(s) I(s) d s
$$


The above results are in cgs units; for MKS units, we multiply the expressions for $w(s), w_{\lambda}(s)$, and $\varkappa$ by $\left(Z_{0} c / 4 \pi\right)$.

In the last bend of $\mathrm{BC} 2$ the bunch can be approximated by a uniform distribution. For a uniform distribution, the bunch wake is simply given by the step-function response, $w_{\lambda}(s)=S(s) / \ell$ [see Eq. 7], with $-\ell \leq s \leq 0$ (the tail is at $s=0)$. The loss factor $\varkappa$ is just the average of this function. We easily see that $\varkappa$ for a uniform distribution is 1.13 times that for a Gaussian distribution of the same rms length, $\sigma_{z}=\ell / \sqrt{12}$. Thus in LCLS-II with $Q=300 \mathrm{pC}$ and a uniform bunch distribution, the power radiated in BX24 is $P_{c s r}=54.8 \mathrm{~W}$.

Performing the integrals using the numerically obtained $I(s)$ (shown in Fig. 2), we find that in the last bend of $\mathrm{BC} 2$ (BX24) the loss factor is $\varkappa=$ $850 \mathrm{~V} /(\mathrm{pC} \mathrm{m})$, compared to $920 \mathrm{~V} /(\mathrm{pC} \mathrm{m})$ for the Gaussian approximation of Table I. Consequently, the power radiated is $P_{c s r}=42.1 \mathrm{~W}$ for the real distribution, compared to the $45.7 \mathrm{~W}$ given for the Gaussian in the table.

\section{$100 \mathrm{pC}$ Case}

For the nominal, $100 \mathrm{pC}$ case we have obtained the bunch shape in L3 and repeated the calculation. The bunch shape and the bunch wake are given in Fig. 3. In this case the current distribution is slightly double-horned, with little tail, and the rms length $\sigma_{z}=7.9 \mu \mathrm{m}$ (the red curve in the figure). The dashed curve gives a uniform approximation, with full width $27.5 \mu \mathrm{m}$ and a peak of $1.09 \mathrm{kA}$.

Performing the integrals using the numerically obtained $I(s)$, we find that in the last bend of $\mathrm{BC} 2$ (BX24) the loss factor is $\varkappa=4300 \mathrm{~V} /(\mathrm{pC} \mathrm{m})$, compared to $4200 \mathrm{~V} /(\mathrm{pC} \mathrm{m})$ for the Gaussian approximation. Consequently, the power radiated is $P_{c s r}=23.9 \mathrm{~W}$ for the real distribution, compared to the $23.4 \mathrm{~W}$ for 


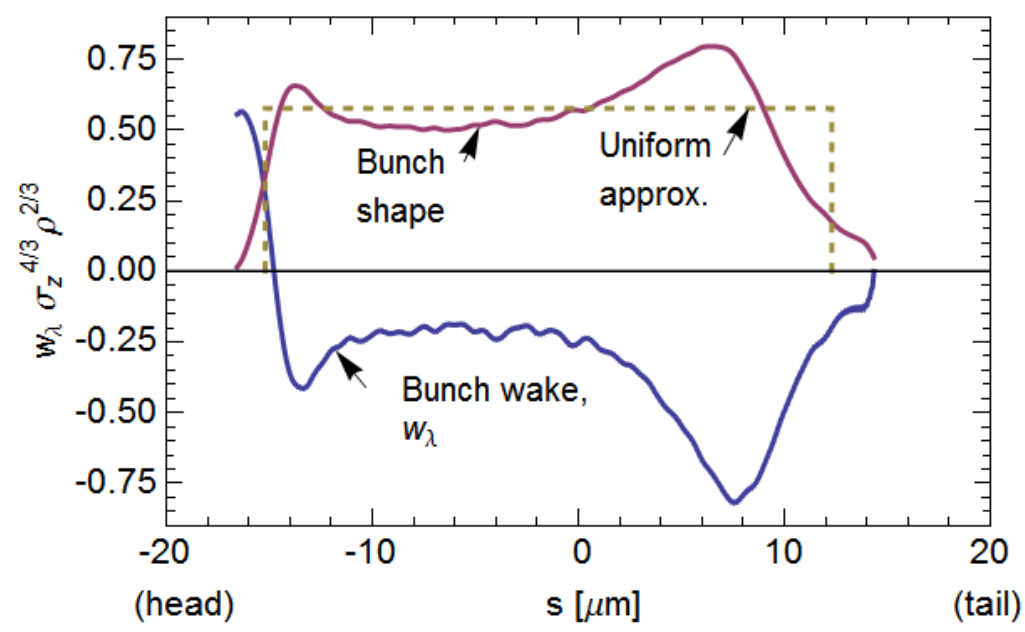

FIG. 3. For the actual $100 \mathrm{pC}$ charge distribution, the bunch wake $w_{\lambda}(s)$ (blue curve) [6]. The bunch shape is also shown, with the head to the left (the red curve). The dashed curve gives a uniform approximation, with full width $27.5 \mu \mathrm{m}$, rms length $7.9 \mu \mathrm{m}$, and peak $1.09 \mathrm{kA}$.

the Gaussian. With the charge and bunch length both reduced by a factor 3 compared to the $300 \mathrm{pC}$ case, we expect the power to scale as $P_{c s r} \sim Q^{2} / \sigma_{z}^{4 / 3} \approx$ $(1 / 3)^{2 / 3} \approx 0.48$ compared to that case; this approximation agrees well with the more accurate calculation.

\section{ACKNOWLEDGEMENTS}

We thank L. Wang for providing us with the bunch shapes used in the Appendix. Work supported by Department of Energy contract DE-AC0276SF00515. 


\section{REFERENCES}

[1] A. Chao and M. Tigner eds., Handbook of accelerator physics and engineering, 3rd Printing (World Scientific Publishing Co., Singapore, 1999) p. 233.

[2] J.B. Murphy, S. Krinsky, and R.L. Gluckstern, Particle Accelerators 57, 9 (1997).

[3] K. Bane, Y. Cai, and G. Stupakov, Phys. Rev. ST Accel. Beams 13, 104402 (2010).

[4] A. Chao and M. Tigner eds., Handbook of accelerator physics and engineering, 3rd Printing (World Scientific Publishing Co., Singapore, 1999) p. 205.

[5] K. Bane and P. Emma, Proc. of 2005 Part. Acc. Conf. in Knoxville, TN, 2005, p. 4266.

[6] L. Wang, private communication. 